\title{
Изучение профиля распределения железа, имплантированного в кремний
}

\author{
(C) А.В. Кожемяко ${ }^{1}$, Ю.В. Балакшин ${ }^{2}$, А.А. Шемухин ${ }^{2}$, В.С. Черныш ${ }^{1,2}$ \\ ${ }^{1}$ Московский государственный университет им. М.В. Ломоносова (физический фракультет), \\ 119991 Москва, Россия \\ ${ }^{2}$ Научно-исследовательский институт ядерной физики им. Д.В. Скобельцына, \\ 119991 Москва, Россия \\ E-mail: anastasiia.kozhemyako@mail.ru
}

(Получена 23 ноября 2016 г. Принята к печати 28 ноября 2016 г.)

Проведена имплантация ионов железа с энергиями 90, 250 кэВ и дозой облучения $10^{16} \mathrm{~cm}^{-2}$ в монокристалл кремния с ориентацией (110). Методом резерфордовского обратного рассеяния в сочетании с каналированием изучены профили распределения внедренной примеси, а также профили распределения радиационных дефектов в кристаллической решетке. Экспериментальные результаты сравниваются с результатами моделирования в программе TRIM. Показано, что при энергии 4.6 кэВ/нуклон средние проективные пробеги совпадают, однако при энергии 1.6 кэВ/нуклон различие составляет $35 \%$. Кроме того показано, что расчет некорректно учитывает дозовую зависимость при энергиях $1.6-4.6$ кэВ/нуклон.

DOI: 10.21883/FTP.2017.06.44556.8460

\section{1. Введение}

Метод ионной имплантации - один из основных методов создания и модификации твердотельных структур. Современный этап развития электронной компонентной базы характеризуется высокой степенью интеграции, для этого требуется создание полупроводниковых структур размерами порядка десятка и единиц нанометров, что соответствует энергии имплантации в диапазоне десятков и сотен кэВ [1-4]. Проектируются и создаются ключевые элементы микросхем, в которых активный элемент реализуется несколькими или даже одним атомом. Были продемонстрированы прототипы одноатомных одноэлектронных устройств: квантового бита [5-7], квантового логического вентиля [8], устройств для квантовой метрологии $[9,10]$, сверхчувствительных зарядовых сенсоров для биологических применений [11]. Точность моделирования распределения внедряемой примеси по глубине будет в значительной мере определять рабочие характеристики элемента микросхемы.

Несмотря на развитую теорию взаимодействия ускоренных заряженных частиц с твердым телом и обширную экспериментальную базу данных для высоких энергий, программы, моделирующие проникновение примеси в мишень, зачастую дают результат, на десятки процентов отличающийся от наблюдаемого в эксперименте [12]. В работах [13-15] при имплантации тяжелых ионов (золото, железо, ксенон) с энергиями 50-70 кэВ в монокристалл и в оксид кремния расхождение между средними проективными пробегами, определенными в эксперименте и рассчитанными с помощью программы SRIM-2013 (TRIM) [16,17], составило от 40 до 50\%, а для энергии 300 кэВ эта разница составила $14 \%$. Также при имплантации тяжелых ионов в углерод с энергиями от 10 до 300 кэВ экспериментальные значения средних проективных пробегов оказались выше рассчитанных в
TRIM на 20-40\% [12]. При имплантации ионов ксенона с энергиями 20-300 кэВ в карбид кремния эта разница составляла от 11 до $20 \%$ [18]. В работе [19] при имплантации ионов ксенона в монокристалл кремния c энергией в диапазоне от 200 до 600 кэВ разница между измеренными средними проективными пробегами и пробегами, рассчитанными в TRIM, оказалась всего 3-8\%. В работе [20] после проведения последовательной имплантации ионов $\mathrm{Ga}^{+}$с энергией 80 кэВ и $\mathrm{N}^{+}$ с энергией 40 кэВ в кремний наблюдалось совпадение экспериментальных проективных пробегов с результатом расчета программы TRIM. Несмотря на указанные расхождения, в некоторых работах результаты расчетов с помощью программы TRIM принимаются за эталон и используются для интерпретации экспериментальных результатов [21]. Таким образом, при низких и средних энергиях для тяжелых ионов наблюдается значительное различие между рассчитанными и экспериментальными распределениями имплантированных частиц, в особенности при энергиях имплантации в несколько кэВ на 1 нуклон.

В данной работе представлены экспериментальные результаты по изучению профилей распределения ионов железа, имплантированных с энергиями 1.6 и 4.6 кэВ/нуклон в монокристалл кремния и возникших при этом дефектов кристаллической структуры мишени. Экспериментальные данные были сопоставлены с расчетами, полученными с помощью программы TRIM. Выбор ионов железа для модификации кремния обусловлен тем, что этот материал обладает перспективными магнитными и оптическими свойства $[15,22,23]$.

\section{2. Методика эксперимента}

Эксперименты по имплантации ионов железа $\left(\mathrm{Fe}^{+}\right)$в монокристаллический кремний $\mathrm{Si}(110)$ проведены при 
энергиях пучка 90 и 250 кэВ на базе ускорительного комплекса тяжелых ионов HVEE-500 в Лаборатории ионно-пучковых нанотехнологий МГУ [24].

Известно, что кристаллическая структура массивных полупроводниковых материалов становится полностью разупорядоченной под воздействием ионного облучения при дозах ионов $10^{16} \mathrm{~cm}^{-2}$ [25]. Так как TRIM считает материал мишени полностью аморфным, то для минимизации влияния упорядоченности структуры мишени на профиль распределения примеси, была выбрана доза ионов $10^{16} \mathrm{~cm}^{-2}$. Плотность тока пучка ионов составляла $80 \mathrm{HA} / \mathrm{cm}^{2}$, что исключало нагрев образца в процессе облучения.

Для устранения каналирования вдоль главных кристаллографических осей облучение проводилось под углом $7^{\circ}$ относительно нормали к поверхности образца. Однородность облучения обеспечивалась сканированием образца пучком в двух взаимно перпендикулярных направлениях. Плотность тока ионов определялась с помощью цилиндров Фарадея, расположенных в экспериментальной камере, что в сочетании с диафрагмами, позволяющими подавить вторичную электронную эмиссию, определяло точность набора дозы. Эксперименты проводились при комнатной температуре и остаточном давлении $5 \cdot 10^{-6}$ мбар.

Степень разрушения кристаллической структуры кремния до и после имплантации, а также профиль распределения внедренной примеси изучались с помощью методики резерфордовского обратного рассеяния (РОР, RBS) в сочетании с каналированием [26] на ускорителе HVEE AN-2500. Энергия анализирующего пучка ионов гелия $\left(\mathrm{He}^{+}\right)$составляла $1.8 \mathrm{MэВ} \mathrm{при} \mathrm{угле} \mathrm{рассе-}$ яния $165^{\circ}$ относительно направления распространения пучка. Каждый образец был исследован в двух режимах: при падении пучка параллельно кристаллографической оси $\langle 110\rangle$, а также в направлении, не содержащем открытых каналов. Направление $\langle 110\rangle$ в кристалле определялось согласно измерению выхода обратно рассеяных ионов при изменении положения образца относительно пучка. Для этого образец устанавливался таким образом, чтобы нормаль к поверхности образца и направление распространения пучка отличались друг от друга на небольшой угол, $\sim(2-4)^{\circ}$. Затем на образец направлялось определенное количество анализирующих частиц и регистрировался сигнал с детектора. Образец на гониометрической системе отклонялся на малый угол, $\sim(0.1-0.2)^{\circ}$, и снова проводился процесс облучения с заданной дозой и регистрацией обратно рассеянных частиц. Отклонение образца на малые углы с последующим облучением осуществлялось до тех пор, пока не была получена характерная кривая зависимости выхода обратно рассеянных частиц от угла между нормалью к поверхности образца и направлением распространения пучка. Сканирование проводилось в двух взаимно перпендикулярных плоскостях. Положение образца, в котором наблюдается наибольший минимум выхода обратно рассеянных частиц, соответствует распространению пучка вдоль главной кристаллографической оси $\langle 110\rangle$.
Расчет проводился с помощью программы TRIM, которая позволяет вычислять профили распределения внедренных частиц и профили распределения дефектов.

\section{3. Результаты и обсуждение}

В начале работы с помощью методики РОР были исследованы исходные образцы монокристалла кремния (рис. 1 , кривые $a$ и $b$ ). Отношение максимума сигнала обратно рассеянных ионов $\mathrm{He}^{+}$в направлении каналирования (кривая $b$ ) к сигналу в направлении, не содержащем открытых каналов (кривая $a$ ), характеризует степень кристалличности образца: чем меньше это отношение, тем лучше кристаллическая структура. В случае совершенного кристалла кремния в результате эффекта каналирования уровень сигнала обратнорассеянных ионов резко уменьшается (до $n=30$ раз), что и видно в спектре $(b)$ (рис. 1). Для используемых в работе монокристаллов с ориентацией (110) было получено уменьшение сигнала в 25 раз. Увеличение выхода обратно рассеянных ионов с уменьшением энергии связано с зависимостью сечения обратного рассеяния от энергии. Характерной особенностью энергетических спектров, снятых в режиме каналирования, является наличие максимумов от присутствующих в образце атомов примеси или атомов, выбитых из узла решетки при ионной имплантации. В исходной структуре вблизи 500-го канала (кривая $b$ ) наблюдается так называемый поверхностный пик, который соответствует естественному окислу. Толщина слоя с разупорядоченными атомами кремния позволяет оценить его примерно в 3 нм.

После облучения монокристалла кремния ионами железа с энергией 90 кэВ в спектрах РОР появился

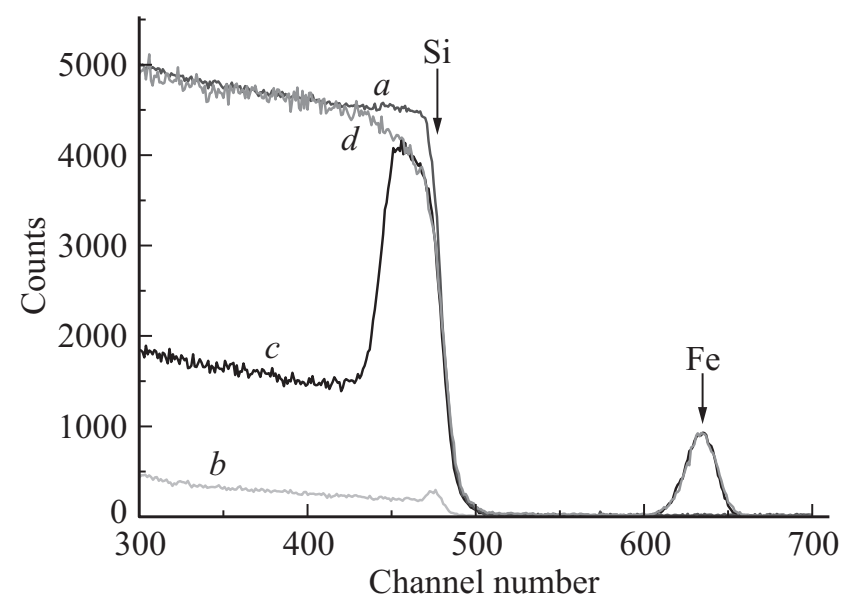

Рис. 1. Энергетические спектры обратно рассеянных ионов $\mathrm{He}^{+}$с энергией $1.8 \mathrm{MэВ} \mathrm{для} \mathrm{угла} \mathrm{рассеяния} 165^{\circ}$ после имплантации ионов $\mathrm{Fe}^{+}$с энергией 90 кэВ. Спектры исходной структуры: $a-$ в направлении, не содержащем открытых каналов; $b-$ в направлении каналирования. Спектры после имплантации: $c-$ в направлении каналирования; $d-$ в направлении, не содержащем открытых каналов. 


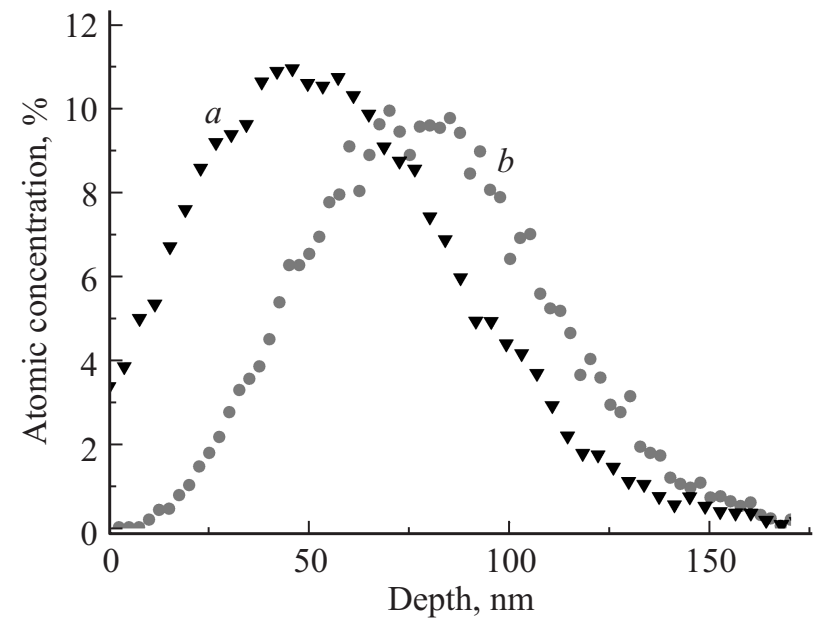

Рис. 2. Профиль распределения по глубине имплантированных ионов $\mathrm{Fe}^{+}$с энергией 90 кэВ: $a$ - экспериментальные данные; $b$ - моделирование с помощью программы TRIM.

дополнительный пик в высокоэнергической части спектра (кривые $c, d)$, соответствующий обратно рассеянным ионам гелия от железа, проникшего при облучении мишени в объем кремния. В спектре, записанном при падении ионов $\mathrm{He}^{+}$в направлении, в котором отсутствуют открытые каналы (кривая $c$ ), вблизи каналов 450-500, соответствующих поверхностным слоям кремния, в отличие от аналогичного спектра для исходной структуры, отсутствует провал. Из этого можно сделать вывод о том, что в рассматриваемой области полностью разрушена кристаллическая структура в поверхностных слоях под действием облучения (кривая $c$ совпадает с кривой $d$ ). Отметим, что спектры РОР от исходной структуры и после ионного облучения (кривые $a$ и $d$ ), полученные в направлении, не содержащем открытых каналов, не совпадают в области каналов 435-475, что говорит о замещении атомов кремния внедренной примесью.

На рис. 2 представлен профиль распределения внедренных ионов железа при энергии имплантации 90 кэВ и дозе $10^{16} \mathrm{~cm}^{-2}$ в увеличенном масштабе (кривая $a$ ). Переход от энергетических каналов к глубине был осуществлен на основании известных значений характеристических потерь [26]. На поверхности присутствуют атомы примеси в концентрации 3\%. Распределение имеет максимум на расстоянии 50 нм от поверхности и ширину на полувысоте 80 нм.

Расчет, проведенный в программе TRIM (кривая $b$ ), дает симметричное распределение со средним проективным пробегом 77 нм и шириной на полувысоте 75 нм. При этом распределение имплантированных атомов начинается на глубине около 10 нм от поверхности. Отметим, что, согласно спектрам РОР, наблюдается замещение атомов мишени внедренной примесью. Однако TRIM не учитывает рекомбинацию межузельных атомов с вакансиями и изменение состава мишени во время имплантации. Оценка учета влияния внедренных ионов осуществлялась следующим образом: в мишень, состоящую на 93\% из кремния и на 7\% из железа, имплантировались ионы железа. В этом случае средний проективный пробег составил 70 нм, при этом распределение стало у́же (ширина на полувысоте составила 54 нм). Таким образом, учет модификации поверхности дает расхождение средних проективных пробегов на 6\% меньшее, чем в случае моделирования процесса имплантации железа в чистый кремний.

Также следует отметить, что расчет дает на 25\% меньшую концентрацию внедренной примеси. В работе [15], где была проведена имплантация монокристалла кремния ионами железа с энергией 50 кэВ и дозой $2.16 \cdot 10^{17} \mathrm{~cm}^{-2}$, напротив, расчет концентрации с помощью TRIM дает значения, превышающие экспериментальные данные более чем в 2 раза.

В следующем эксперименте была проведена имплантация ионов железа с энергией 250 кэВ и дозой $10^{16} \mathrm{~cm}^{-2}$ (рис. 3). Для сравнения приведен спектр РОР от исходной подложки в направлении, не содержащем открытых каналов (кривая $a$ ). Видно, что после облучения присутствует спад в районе каналов 400-500 (кривая $b$ ). Уменьшение в приповерхностном слое концентрации кремния, вероятнее всего, произошло в результате замещения атомов кремния внедренными ионами железа. В отличие от рис. 1, где после имплантации $\mathrm{Fe}^{+}$с энергией 90 кэВ произошло полное разупорядочение, после имплантации ионов с энергией 250 кэВ кривые $b$ и $c$ не совпадают, т. е. в этом случае в приповерхностных слоях монокристалла кремния на глубину пробега ионов $\mathrm{Fe}^{+}$ полного разупорядочения не произошло.

Профиль распределения имплантированных ионов $\mathrm{Fe}^{+}$представлен на рис. 4 в увеличенном масштабе (кри-

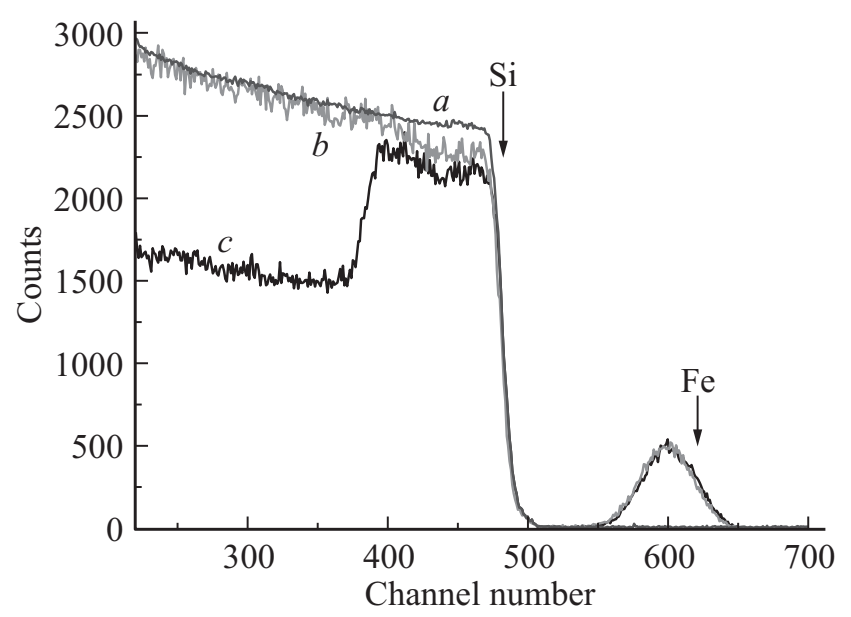

Рис. 3. Энергетические спектры обратно рассеянных ионов $\mathrm{He}^{+}$с энергией $1.8 \mathrm{MэВ} \mathrm{для} \mathrm{угла} \mathrm{рассеяния} 165^{\circ}$ после имплантации ионов $\mathrm{Fe}^{+}$с энергией 250 кэВ. Спектр исходной структуры: $a-$ в направлении, не содержащем открытых каналов. Спектры после имплантации: $b-$ в направлении, не содержащем открытых каналов; $c-$ в направлении каналирования. 
вая $a$ ). Максимум распределения находится на расстоянии 220 нм от поверхности. Согласно программе TRIM (кривая $b$ ), средний проективный пробег $\mathrm{Fe}^{+}$с энергией 250 кэВ в кремниевой матрице составляет 210 нм. Распределения имеют симметричную форму. Ширины экспериментального и расчетного распределений на полувысоте составили 170 нм. Для этой энергии мы видим хорошее согласие экспериментальных данных и данных, полученных с помощью моделирования. Однако расчет с помощью программы TRIM дает заниженную на 30\% концентрацию внедренной примеси.

Сравнение распределения дефектов, определенных экспериментально (кривая $a$ ), с профилем дефектов, рассчитанным TRIM (кривая $b$ ) для энергии пучка 90 кэВ, приведено на рис. 5. Параметр аморфизации по РОР показывает отношение сигналов спектров, снятых в

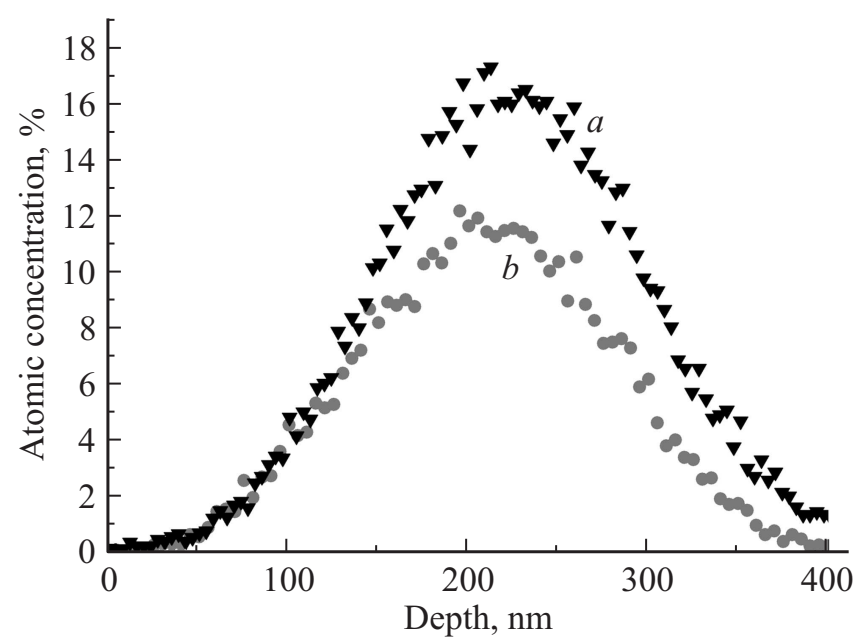

Рис. 4. Профиль распределения по глубине имплантированных ионов $\mathrm{Fe}^{+}$с энергией 250 кэВ: $a$ - экспериментальные данные; $b$ - моделирование с помощью программы TRIM.

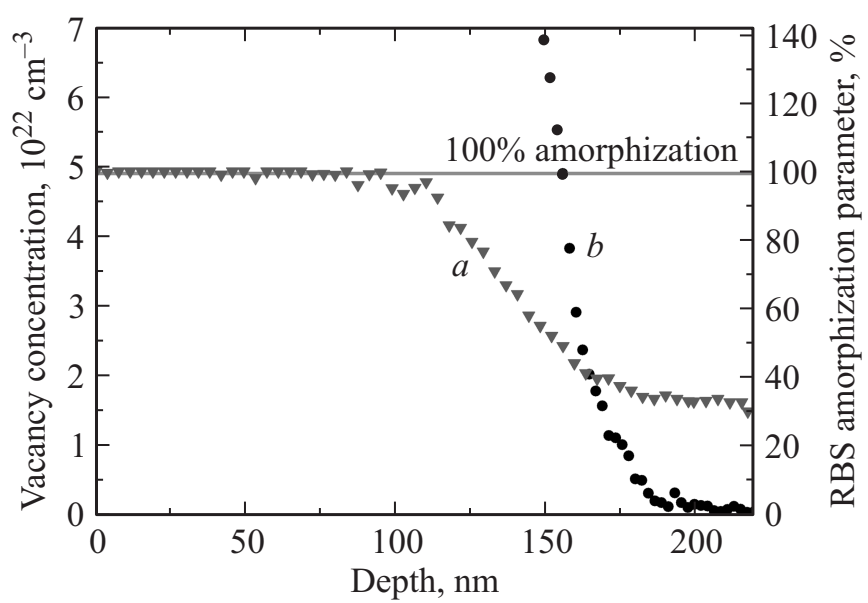

Рис. 5. Профиль распределения дефектов, образованных после облучения ионами $\mathrm{Fe}^{+}$с энергией 90 кэВ: $a-$ экспериментальные данные; $b-$ моделирование с помощью программы TRIM.

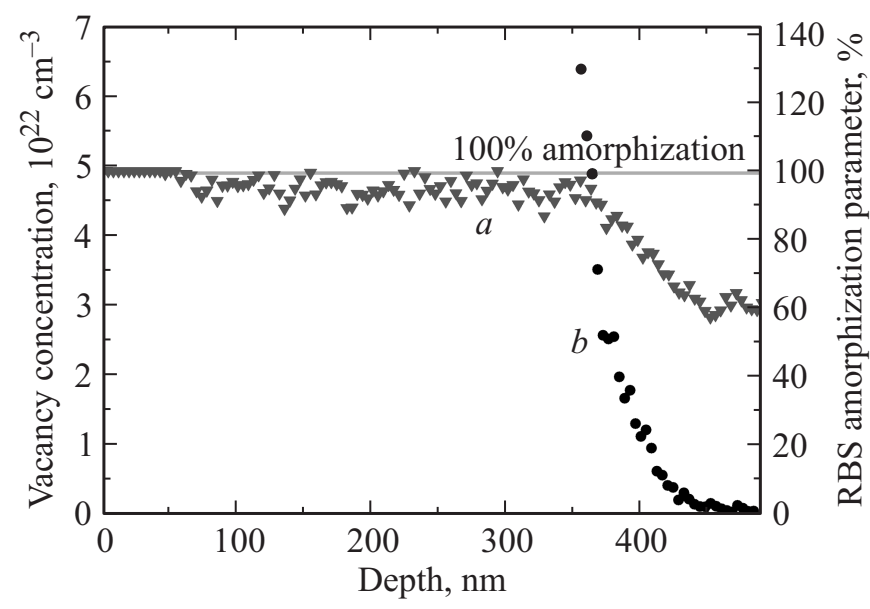

Рис. 6. Профиль распределения дефектов, образованных после облучения ионами $\mathrm{Fe}^{+}$с энергией 250 кэВ: $a-$ экспериментальные данные; $b-$ моделирование с помощью программы TRIM.

направлении каналирования и в направлении, не содержащем открытых каналов. При сравнении с теоритическими расчетами авторы считали монокристалл кремния полностью разупорядоченным, когда число смещений равно числу атомов в слое, т.е. полностью разупорядоченная область в экспериментальном распределении дефектов соответствует концентрации вакансий $4.9 \cdot 10^{22} \mathrm{~cm}^{-3}$ теоретического распределения (показано сплошной линией). Согласно теоретическим расчетам, такое количество смещений наблюдается на глубине 155 нм. Однако по экспериментальным данным видно, что аморфизация наблюдается только до глубины 110 нм и далее степень разупорядочения падает до $35 \%$ на глубине 180 нм от поверхности. Отсутствие падения выхода обратно рассеянных частиц до уровня исходной структуры в режиме каналирования объясняется деканалированием пучка ионов гелия по мере его прохождения в глубь кристалла через разрушенную ионным облучением область.

На рис. 6 приведено сравнение профиля распределения дефектов, определенного экспериментально (кривая $a$ ), с профилем дефектов, рассчитанным TRIM (кривая $b$ ) для энергии пучка имплантированных ионов 250 кэВ. Согласно экспериментальной кривой, аморфизация произошла только на глубине до 54 нм и вплоть до глубины 360 нм средняя разупорядоченность по РОР составляла 95\%. По расчетам же аморфизация происходит на глубину до 365 нм. Кроме того, видно, что при указанных параметрах эксперимента образования дефектов глубже 450 нм не происходит. Однако, согласно экспериментальным данным, на глубине 450 нм параметр аморфизации по РОР составляет $60 \%$. В этом случае, в отличие от рис. 5 , деканалированных частиц больше, так как модифицированный ионным облучением слой толще. 


\section{4. Заключение}

Методом резерфордовского обратного рассеяния в сочетании с каналированием изучены профили распределения железа, имплантированного в кремний. При облучении с энергией 90 кэВ различие экспериментальных и рассчитанных средних проективных пробегов составляет $35 \%$. С увеличением энергии имплантации до 250 кэВ профили распределения внедренных частиц, полученные с помощью POP и TRIM, находятся в хорошем согласии. Установлено, что при используемых параметрах имплантации моделирование TRIM дает заниженную на 25-30\% оценку концентрации внедренной примеси по отношению к экспериментальным данным.

Анализ образовавшихся дефектов при энергии 1.6 кэВ/нуклон показывает, что TRIM дает завышенную на 30\% оценку. Согласно экспериментальным данным, с увеличением энергии до 4.6 кэВ/нуклон полного разупорядочения кристаллической структуры кремния на глубину проникновения ионов железа не происходит. Однако расчет при указанных параметрах имплантации в программе TRIM показывает образование значительно большего количества вакансий.

Дальнейшее исследование профилей распределения по глубине внедренной примеси и радиационных дефектов позволит как решить ряд задач прикладного направления, так и получить дополнительную информацию о фундаментальном характере взаимодействия ускоренных заряженных частиц в веществе.

Исследование выполнено за счет гранта Российского научного фонда (проект № 16-12-00072).

\section{Список литературы}

[1] Y. Zhang, H. Huang, D. Bi, M. Tang, Z. Zhang. Nucl. Instr. Meth. B, 319, 141 (2014).

[2] B. Zheng, N. Iketa, Y. Takeda, K. Sato, R. Sato, H. Amekura, K. Oyoshi, M. Song, D. Ila, N. Kishimoto. Nucl. Instr. Meth. B, 272, 183 (2010).

[3] А.А. Шемухин, А.В. Назаров, Ю.В. Балакшин, В.С. Черныш. Поверхность. Рентген., синхротрон. и нейтрон. исслед., № 3, 56 (2014).

[4] A.A. Shemukhin, A.V. Nazarov, Yu.V. Balakshin, V.S. Chernysh. Nucl. Instr. Meth. B, 354, 274 (2015).

[5] B.E. Kane. Nature, 393, 133 (1998).

[6] J.J. Pla, K.Y. Tan, J.P. Dehollain, W.H. Lim, J.J. Morton, D.N. Jamieson, A.S. Dzurak, A. Morello. Nature, 489, 541 (2012).

[7] M. Fuechsle, J.A. Miwa, S. Mahapatra, H. Ryu, S. Lee, O. Warschkow, L.C. Hollenberg, G. Klimeck, M.Y. Simmons. Nat. Nanotechnol., 7, 242 (2012).

[8] M. Veldhorst, C.H. Yang, J.C.C. Hwang, W. Huang, J.P. Dehollain, J.T. Muhonen, S. Simmons, A. Laucht, F.E. Hudson, K.M. Itoh, A. Morello, A.S. Dzurak et al. Nature, 526, 410 (2015).

[9] G. Yamahata, K. Nishiguchi, A. Fujiwara. Nat. Commun., 5, 1 (2014).

[10] G.C. Tettamanzi, R. Wacquez, S. Rogge. New J. Phys., 16, 63036 (2014).
[11] D.E. Presnov, V.V. Shorokhov, S.V. Amitonov, A.S. Trifonov, V.A. Krupenin. The 8th General Meeting of ACCMS-VO (Tohoku University, Japan, 2013) PS-3.

[12] P.L. Grande, F.C. Zawislak, D. Fink, M. Behar. Nucl. Instr. Meth. B, 61, 282 (1991).

[13] P.L. Grande, P.F.P. Fichtner, M. Behar, F.C. Zawislak. Nucl. Instr. Meth. B, 35, 17 (1988).

[14] A. Naas, E. Ntsoenzok, D. De Sousa-Meneses, B. Hakim, A. Beya-Wakata. Nucl. Instr. Meth. B, 339, 46 (2014).

[15] W.J. Lakshantha, V.C. Kummari, T. Reinert, F.D. McDaniel, B. Rout. Nucl. Instr. Meth. B, 332, 33 (2014).

[16] J.F. Ziegler, M.D. Ziegler, J.P. Biersack. Nucl. Instr. Meth. B, 268, 1818 (2010).

[17] J.F. Ziegler, J.P. Biersack, U. Littmark. The Stopping and Range of Ions in Matter (Pergamon, N.Y., 1985).

[18] A. Föhl, R.M. Emrick, H.D. Carstanjen. Nucl. Instr. Meth. B, 65, 335 (1992).

[19] T. Chunyu, X. Yueyuan, Y. Hong, S. Xiufang. Nucl. Instr. Meth. B, 42, 1 (1989).

[20] Д.С. Королев, А.Н. Михайлов, А.И. Белов, В.К. Васильев, Д.В. Гусейнов, Е.В. Окулич, А.А. Шемухин, А.В. Пирогов, Д.А. Павлов, Д.И. Тетельбаум, С.И. Суродин, Д.Е. Николичев, А.В. Нежданов. ФТП, 50, 274 (2016).

[21] L. Himics, S. Tóth, M. Veres, A. Tóth, M. Koós. Appl. Surf. Sci., 328, 577 (2015).

[22] W.J. Lakshantha, M.S. Dhoubhadel, T. Reinert, F.D. McDaniel, B. Rout. Nucl. Instr. Meth. B, 365, 114 (2015).

[23] M. Ishimary. Nucl. Instr. Meth. B, 258, 490 (2007).

[24] А.А. Шемухин, П.Н. Черных, В.С. Черныш, Ю.В. Балакшин, А.В. Назаров. Прикладная физика, № 5, 59 (2013).

[25] А.А. Шемухин, Ю.В. Балакшин, П.Н. Черных, В.С. Черныш. Поверхность. Рентген., синхротрон. и нейтрон. исслед., № 4, 25 (2013).

[26] M. Nastasi, J.W. Mayer, Y. Wang. Ion beam analysis, fundamentals and applications (Boca Raton, FL, CRC Press, 2015).

Редактор Л.В. Шаронова

\section{The study of implanted in silicon iron distribution profile}

\author{
A.V. Kozhemiako ${ }^{1}$, Y.V. Balakshin ${ }^{2}$, A.A. Shemukhin ${ }^{2}$, \\ V.S. Chernysh ${ }^{1}$ \\ ${ }^{1}$ Moscow State University \\ (Faculty of Physics), \\ 119991 Moscow, Russia \\ 2 Skobeltsyn Institute of Nuclear Physics, \\ Moscow State University, \\ 119991 Moscow, Russia
}

Abstract Iron ions have been implanted with energies of 90 , $250 \mathrm{keV}$ and a dose of $10^{16} \mathrm{~cm}^{-2}$ in a single crystal silicon with the orientation (110). The distribution profiles of the introduced impurity were studied with the Rutherford backscattering in combination with channeling, and the distribution profiles of radiation defects in the crystal lattice were studied as well. The experimental results were compared with the results of simulation program TRIM. It has been shown that at the energy of $3 \mathrm{keV} /$ nucleon the average projected ranges are the same, however at the energy of $1 \mathrm{keV} /$ nucleon the difference is $35 \%$. In addition, it is shown that the calculation does not correctly account for the dose dependence at energies $1-3 \mathrm{keV} /$ nucleon. 